\title{
Case Study: Enhanced Clustering Technique on Sequential Data Streams using Optics and Chameleon
}

\author{
K. SanthiSree, PhD \\ Professor \\ Jawaharlal Nehru \\ Technological University \\ Hyderabad
}

\author{
V. Vineela \\ Student \\ Jawaharlal Nehru \\ Technological University \\ Hyderabad
}

\author{
Y. Ambica \\ Assistant Professor(c), \\ Jawaharlal Nehru \\ Technological University \\ Hyderabad
}

\author{
Ch. Anitha \\ Assistant Professor(c), \\ Jawaharlal Nehru \\ Technological University \\ Hyderabad
}

\begin{abstract}
Huge data is getting accumulated every second in the real world .Clustering on web usage data is useful to identify what users are exactly looking for on the world wide web, like user traversals, users behavior and their characteristics, which helps for Web personalization. Clustering web sessions is to group them based on similarity and consists of minimizing the Intra-cluster similarity and maximizing the Inter-group similarity. In the past there exist multiple similarity measures like Euclidean, Jaccard ,Cosine , Manhattan, Minkowski, and many to measure similarity between web patterns. In this paper, we enhanced Chameleon Clustering Algorithm(CCA) based on CHAMELEON. Experiments are performed on MSNBC.COM website (free online news channel), on sequential data streams in the context of clustering in the domain of Web usage mining. Clustering in data mining is a discovery process that groups a set of data such that the intra-cluster similarity is maximized and the inter-cluster similarity is minimized. Existing clustering algorithms, such as K-means, PAM, CLARANS, DBSCAN, CURE, and ROCK are designed to find clusters that fit some static models Specially, we present a detailed comparison of OPTICS and CHAMELEON and the results illustrate that CHAMELEON is much more suitable for clustering the dynamic datasets. The Inter-cluster and Intra-cluster distances are computed using Average Levenshtein Distance (ALD) to demonstrate the usefulness of the proposed approach in the context of web usage mining. This new enhanced (CHAMELEON algorithm)has good results when compared with existing OPTICS clustering technique, and provided good time requirements of the newly developed algorithms.
\end{abstract}

\section{Keywords}

Sequence Mining, Clustering, Density Based Clustering(optics). Data Mining, Clustering, similarity measures, Web Personalization.

\section{INTRODUCTION}

\subsection{Clustering}

Clustering is a process of categorizing the data into multiple clusters where all the patterns lying in one cluster are similar to one another and dissimilar when compared to the patterns lying in the other cluster. Different types of clustering techniques are Partitioning ,Hierarchical, Density-based ,Grid-based and Model-Based algorithms. Types of Density based clustering techniques are DBSCAN, Optics and Denclue. Hierarchical clustering algorithms produce a nested sequence of clusters, with a single all-inclusive cluster at the top and single point clusters at the bottom. In the single link method [JD88], each cluster is represented by all the data points in the cluster.
The similarity between two clusters is measured by the similarity of the closest pair of data points belonging to different clusters. Unlike the centroid/medoid based methods, this method can find clusters of arbitrary shape and different sizes. Here in this work, we are concentrating on Hierarchical clustering technique.

\subsection{Similarity Measures}

Similarity measures are used to find out how similar are two sequences are. In the history many similarity measures exist, and they are Euclidean, Jaccard, Cosine, Manhattan and Minkowski measures. These similarity measures are either vector based or frequency based. The Euclidean distance between sequences $\mathrm{S}_{1}=\left(p_{1}, p_{2}, \ldots, p_{n}\right) \quad$ and $\mathrm{S}_{2}=\left(q_{1}, q_{2}, \ldots, q_{n}\right)$ is defined as

$$
\begin{aligned}
& \operatorname{Sim}\left(S_{1}, S_{2}\right)= \\
& \sqrt{\left(S_{1_{1}}-S_{2_{1}}\right)^{2}+\left(S_{1_{2}}-S_{2_{2}}\right)^{2}+\cdots+\left(S_{1_{n}}-S_{2_{n}}\right)^{2}}= \\
& \sqrt{\sum_{i=1}^{n}\left(S_{1_{i}}-S_{2_{i}}\right)^{2}} \text { (Eqn.1) }
\end{aligned}
$$

Jaccard similarity measure is defined as the ratio of the intersection of items between the two sequences to the union of items of the two sequences.

$$
\left(\operatorname{Sim}\left(S_{1}, S_{2}\right)\right)=\frac{\boldsymbol{S}_{\mathbf{1}} \boldsymbol{S}_{\mathbf{2}}}{\left|\boldsymbol{S}_{\mathbf{1}}\right|^{2}+\left|\boldsymbol{S}_{\mathbf{2}}\right|^{2}-\boldsymbol{S}_{\mathbf{1}} \boldsymbol{S}_{\mathbf{2}}}
$$

(Eqn.2)

Cosine similarity measure is the angle between two vectors. The cosine measure is given by

$$
(\operatorname{Eqn} .3) \operatorname{Sim}\left(S_{1}, S_{2}\right)=\frac{\sum_{i=1}^{n}\left(\boldsymbol{S}_{\mathbf{1}} \times \boldsymbol{S}_{\mathbf{2}}\right)}{\sqrt{\sum_{\boldsymbol{i = 1} \mathbf{1}}^{n}\left(\boldsymbol{S}_{\mathbf{1}_{i}}\right)^{\mathbf{2}} \times \sqrt{\sum_{\boldsymbol{i}=\mathbf{1}}^{n}\left(\boldsymbol{S}_{2_{\boldsymbol{i}}}\right)^{2}}}}
$$

\section{EXISTING METHODOLOGY}

In the existing work, the sequences are converted to intermediate representations and the similarity between any two sequences is calculated using any of the similarity measures like Euclidean. OPTICS clustering technique can be applied for clustering. While computing similarity between sequences they either consider the content /information or the order information. 
Algorithm :OPTICS(DB, Eps, MinPts)

Input: $A$ database $D$ with $\mathrm{N}$ samples.

\{Dataset D with $\mathrm{N}$ objects, epsilon(eps) the

radius, and $\mathrm{min}$ pts, i.e.. the number of

minimum points and $\mathrm{C}$ the cluster $\}$

Output: set of Clusters $C=\{c 1, c 2, c 3 \ldots c n\}$

Method:

Step 1: For each point $\mathrm{P}$ of $\mathrm{DB}$

Step 2:N = regionQuery (P, eps).

Step 3: If sizeof(N) < MinPts, mark $P$ as NOISE.

Step4: If $\mathrm{N}>=$ MinPts, then mark $\mathrm{p}$ as core object

Step 5:Add $P$ to the priority queue.

Step 6; Repeat steps 1,2,3,4 until end of the

database DB has reached

\section{Fig 1. Algorithm for Optics Clustering}

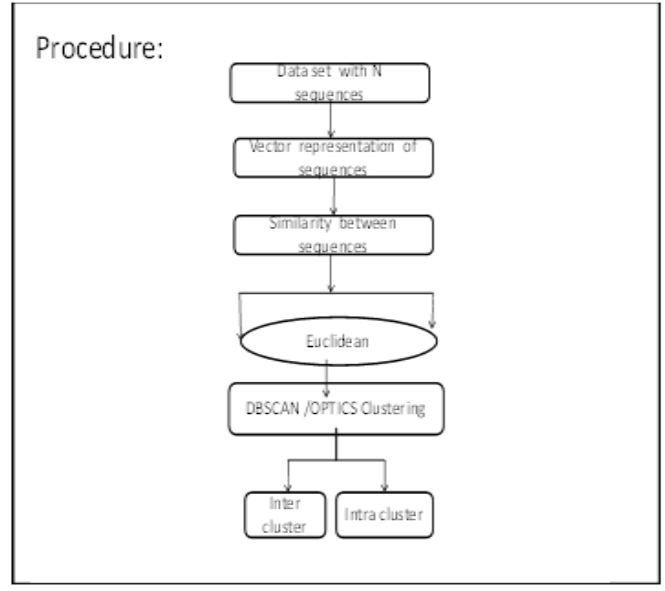

Fig 2. Existing Work Procedure

Example:

Step1: Consider a set of 10 sample sequences (Transactions) randomly from the MSNBC dataset. In the current work, the sequences has to be converted to vector representations. The entire set contains multiple categories of news like \{on-air, misc, news, sports, bbs, front page, local, weather, travel, opinion, msn-news, business etc $\}$. In each sequence presence of category of news is taken as 1 and absence as 0.The vector representation of the sequences is in Table 1 and 2 the rows indicate the transactions $\{\mathrm{T} 1, \mathrm{~T} 2, \mathrm{~T} 3, \mathrm{~T} 4, \mathrm{~T} 5, \mathrm{~T} 6, \mathrm{~T} 7, \mathrm{~T} 8, \mathrm{~T} 9, \mathrm{~T} 10\}$ and the columns indicate the category id i.e news id. In the first sequence for example, on-air is present, misc is present so the particular category id represented as 1 and remaining categories are taken as 0 .

Table 1. Vector Representation Of Sequences

\begin{tabular}{|c|c|c|c|c|c|c|c|c|c|c|c|c|}
\hline $\begin{array}{c}\text { Transacti } \\
\text { on } \times \\
\text { category } \\
\text { id }\end{array}$ & 1 & 2 & 3 & 4 & 5 & 6 & 7 & 8 & 9 & 1 & 1 & 1 \\
\hline T1 & 1 & 1 & 0 & 0 & 0 & 0 & 0 & 0 & 0 & 0 & 0 & 0 \\
\hline T2 & 0 & 0 & 1 & 1 & 0 & 0 & 1 & 0 & 1 & 0 & 0 & 0 \\
\hline T3 & 0 & 0 & 0 & 0 & 1 & 0 & 0 & 0 & 0 & 0 & 0 & 0 \\
\hline
\end{tabular}

\begin{tabular}{|c|c|c|c|c|c|c|c|c|c|c|c|c|}
\hline T4 & 0 & 0 & 1 & 1 & 0 & 1 & 1 & 0 & 0 & 0 & 0 & 0 \\
\hline T5 & 1 & 0 & 0 & 1 & 0 & 0 & 0 & 1 & 0 & 0 & 0 & 0 \\
\hline T6 & 1 & 0 & 0 & 0 & 1 & 0 & 0 & 0 & 1 & 0 & 0 & 0 \\
\hline T7 & 0 & 0 & 1 & 0 & 1 & 1 & 0 & 0 & 0 & 0 & 0 & 0 \\
\hline T8 & 0 & 0 & 0 & 0 & 1 & 1 & 0 & 0 & 0 & 0 & 0 & 0 \\
\hline T9 & 0 & 0 & 1 & 0 & 0 & 0 & 0 & 0 & 1 & 1 & 1 & 0 \\
\hline T10 & 0 & 0 & 1 & 0 & 1 & 1 & 0 & 0 & 0 & 0 & 0 & 1 \\
\hline
\end{tabular}

In Table 2, For example, consider the first sequence/Transaction, on-air is present, whose frequency is 2 , misc is present and its frequency is 4 .so the particular category id 1 and 2 are represented with its frequency 2 and 4 respectively and remaining category id's are considered as 0 .

Table 2. Frequency Representation Of Sequences

\begin{tabular}{|c|c|c|c|c|c|c|c|c|c|c|c|c|}
\hline $\begin{array}{c}\text { Transacti } \\
\text { on } \\
\begin{array}{c}\text { Category } \\
\text { id }\end{array}\end{array}$ & 1 & 2 & 3 & 4 & 5 & 6 & 7 & 8 & 9 & 1 & 1 & 1 \\
\hline T1 & 2 & 4 & 0 & 0 & 0 & 0 & 0 & 0 & 0 & 0 & 0 & 0 \\
\hline T2 & 0 & 0 & 0 & 0 & 3 & 1 & 0 & 0 & 0 & 0 & 0 & 0 \\
\hline T3 & 0 & 0 & 0 & 0 & 0 & 6 & 0 & 0 & 0 & 0 & 0 & 0 \\
\hline T4 & 0 & 0 & 2 & 1 & 0 & 2 & 1 & 0 & 0 & 0 & 0 & 0 \\
\hline T5 & 1 & 0 & 0 & 1 & 0 & 0 & 0 & 3 & 0 & 0 & 0 & 0 \\
\hline T6 & 4 & 0 & 0 & 0 & 1 & 0 & 0 & 0 & 1 & 0 & 0 & 0 \\
\hline T7 & 0 & 0 & 1 & 0 & 2 & 3 & 0 & 0 & 0 & 0 & 0 & 0 \\
\hline T8 & 0 & 0 & 0 & 0 & 1 & 5 & 0 & 0 & 0 & 0 & 0 & 0 \\
\hline T9 & 0 & 0 & 2 & 0 & 0 & 0 & 1 & 2 & 1 & 0 & 0 & 0 \\
\hline T10 & 0 & 0 & 2 & 0 & 1 & 2 & 0 & 0 & 0 & 0 & 1 & 0 \\
\hline
\end{tabular}

Table 3.Sequence similarity matrix using Euclidean Measure

\begin{tabular}{|c|c|c|c|c|c|c|c|c|c|c|}
\hline & & & & & & & & & $\mathrm{T}$ & $\mathrm{T}$ \\
& $\mathrm{T} 1$ & $\mathrm{~T} 2$ & $\mathrm{~T} 3$ & $\mathrm{~T} 4$ & $\mathrm{~T} 5$ & $\mathrm{~T} 6$ & $\mathrm{~T} 7$ & $\mathrm{~T} 8$ & 9 & 0 \\
\hline $\mathrm{T}$ & & 2. & 2. & 2. & 73 & 41 & 2. & & 4 & 2 \\
1 & - & 44 & 23 & 44 & 2 & 4 & 23 & 2 & 4 & 3 \\
\hline $\mathrm{T}$ & 2. & & 2. & 41 & 2. & 2. & 2. & 2. & & 4 \\
2 & 44 & - & 23 & 4 & 23 & 23 & 23 & 23 & 2 & 4 \\
\hline & & & & & & 1. & 1. & & 2. & 1. \\
$\mathrm{~T}$ & 2. & 2. & & 2. & & 41 & 41 & & 2 & 7 \\
3 & 23 & 23 & - & 23 & 2 & 4 & 4 & 1 & 3 & 3 \\
\hline $\mathrm{T}$ & 2. & 1. & 2. & - & 2. & 2. & & & 2. & 2 \\
4 & 44 & 41 & 23 & - & 23 & 64 & 2 & 2 & 4 & 2 \\
\hline
\end{tabular}




\begin{tabular}{|c|c|c|c|c|c|c|c|c|c|c|} 
& & 4 & & & & & & & 4 & \\
\hline & 1. & & & & & & & & 2. & 2. \\
$\mathrm{~T}$ & 73 & 2. & & 2. & & & 2. & & 6 & 6 \\
5 & 2 & 23 & 2 & 23 & - & 2 & 44 & 2 & 4 & 4 \\
\hline & 1. & & 1. & & & & & 1. & 2. & 2. \\
$\mathrm{~T}$ & 41 & 2. & 41 & 2. & & & & 73 & 4 & 4 \\
6 & 4 & 23 & 4 & 64 & 2 & - & 2 & 2 & 4 & 4 \\
\hline & & & 1. & & & & & & 2. & \\
$\mathrm{~T}$ & 2. & 2. & 41 & & 2. & & & & 4 & \\
7 & 23 & 23 & 4 & 2 & 44 & 2 & - & 1 & 4 & 1 \\
\hline $\mathrm{T}$ & & 2. & & & & 7. & & & 2. & 1. \\
8 & 2 & 23 & 1 & 2 & 2 & 2 & 1 & - & 4 & 1 \\
\hline $\mathrm{T}$ & 2. & & 2. & 2. & 2. & 2. & 2. & 2. & & 4 \\
9 & 44 & 2 & 23 & 44 & 64 & 44 & 44 & 44 & - & 4 \\
\hline $\mathrm{T}$ & & & & & & & & & 2. & \\
1 & 2. & 2. & 1. & & 2. & 2. & & 1. & 4 & \\
0 & 23 & 44 & 73 & 2 & 64 & 44 & 1 & 41 & 4 & - \\
\hline
\end{tabular}

In Table 3 indicates a $\mathrm{N} \times \mathrm{N}$ Similarity matrix is calculated where rows and columns indicate the Transactions $\{\mathrm{T} 1, \mathrm{~T} 2, \mathrm{~T} 3, \mathrm{~T} 4, \mathrm{~T} 5, \mathrm{~T} 6, \mathrm{~T} 7, \mathrm{~T} 8, \mathrm{~T} 9, \mathrm{~T} 10\} . \quad$ For example similarity $(\mathrm{T} 1, \mathrm{~T} 2)=2.44$, i.e, similarity between the two sequences $\mathrm{T} 1, \mathrm{~T} 2$ is 2.44 . If the two sequences say $\mathrm{T} 1, \mathrm{~T} 2$ are similar, the $\operatorname{similarity}(\mathrm{T} 1, \mathrm{~T} 2)=0$. If they are more dissimilar, the similarity ratio increases. For example, the similarity between the sequences $(\mathrm{T} 1, \mathrm{~T} 5)=1.732$, which means the two sequences seem to be more similar. The similarity between the sequences $(\mathrm{T} 5, \mathrm{~T} 9)=2.64$, which shows the two sequences seems to be more dissimilar.

Step 4: Applying OPTICS clustering algorithm:

Clusters formed are

- $\mathrm{C} 1=\{\mathrm{T} 3, \mathrm{~T} 5, \mathrm{~T} 6, \mathrm{~T} 8\}$

- $\mathrm{C} 3=\{\mathrm{T} 1, \mathrm{~T} 5, \mathrm{~T} 6, \mathrm{~T} 7, \mathrm{~T} 8, \mathrm{~T} 9\}$

- $\mathrm{C} 5=\{\mathrm{T} 1, \mathrm{~T} 3, \mathrm{~T} 6\}$

- $\mathrm{C} 6=\{\mathrm{T} 1, \mathrm{~T} 3, \mathrm{~T} 5, \mathrm{~T} 7, \mathrm{~T} 8\}$

- $\quad \mathrm{C} 7=\{\mathrm{T} 3, \mathrm{~T} 6, \mathrm{~T} 8, \mathrm{~T} 10\}$

- $\mathrm{C} 9=\{$ NOISE $\}$

- $\mathrm{C} 2=\{$ NOISE $\}$

- $\mathrm{C} 8=\{\mathrm{T} 1, \mathrm{~T} 3, \mathrm{~T} 4, \mathrm{~T} 6, \mathrm{~T} 7\}$ and

- $\mathrm{C} 10=\{\mathrm{T} 3, \mathrm{~T} 7, \mathrm{~T} 8\}$

Applying ICA clustering algorithm, the clusters formed are

- $\mathrm{C} 1=\{\mathrm{T} 1, \mathrm{~T} 3, \mathrm{~T} 5, \mathrm{~T} 6, \mathrm{~T} 8, \mathrm{~T} 7,10\}$

- $\mathrm{C} 2=\{\mathrm{T} 2\}$

- $\mathrm{C} 3=\{\mathrm{T} 4\}$

\section{PROPOSED WORK PROCEDURE}

\subsection{Enhanced Chameleon Clustering} Algorithm(CCA):

The work concentrates on Clustering techniques on data streams in the domain of web usage data .Euclidean similarity measure is used to measure similarity/distance between two sequences and experiments are conducted on various clustering techniques using Optics, and CCA .In all the experiments the running time of the new algorithm (CCA) is best compared to the earlier similarity measures. Figure 3 shows the proposed framework.

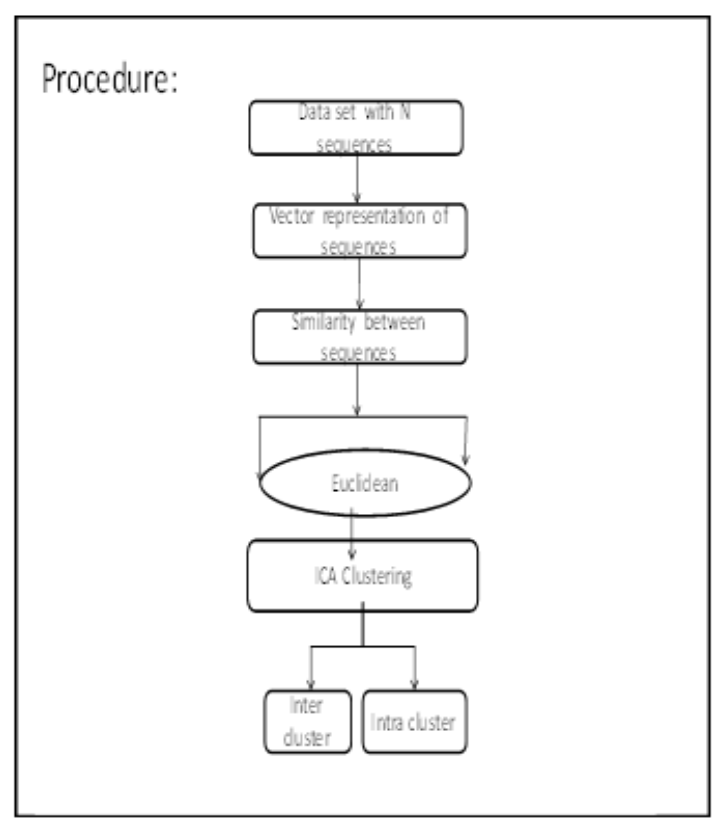

Fig 3: Proposed Work Procedure

\section{EXPERIMENTAL RESULTS}

\subsection{Web Navigation Dataset for Testing}

MSNBC is a famous online news website with has different news subjects. There are 17 categories of newslikefrontpage,news,tech,local,opinion,onair,weather,healt h,living,business,sports,summary,bbs,travelmisc,msn-news, and msn-sports. Web Navigational dataset is considered in Table 4.

Table 4.Web Navigational Dataset

\begin{tabular}{|c|c|}
\hline Sequence & \\
\hline T1 & on-air, misc, misc, misc, on-air, misc \\
\hline T2 & News, sports, tech, local,sports, sports \\
\hline T3 & Sports, bbs, bbs, bbs, bbs, bbs, bbs \\
\hline T4 & Frontpage, frontpage, sports, news, news, local \\
\hline T5 & on-air,weather,weather,weather, sports,sports \\
\hline T6 & on-air, on-air, on-air, on-air, tech, bbs \\
\hline T7 & Frontpage,bbs,bbs,frontpage, frontpage, news \\
\hline T8 & Frontpage,frontpage,frontpage, frontpage, \\
& frontpage, bbs \\
\hline T9 & News, news, travel, opinion, opinion, msn-news \\
\hline T10 & Frontpage, business, frontpage, news news, bbs \\
\hline
\end{tabular}

\subsection{Optics And CCA Experiments on Standard web Navigational Dataset.}

Considered transactions of varying sizes of 5000, $10000,20,000,30000,40000$ from MSNBC dataset. Table 5 
shows the number of clusters formed by applying the existing Optics and proposed ICA. Using the similarity measure like Euclidean, Inter cluster similarity and Intra cluster similarity are calculated.

Table 5. Inter and Intra cluster distance for OPTICS and CCA

\begin{tabular}{|c|c|c|c|c|c|}
\hline \multicolumn{6}{|c|}{ OPTICS -Clustering Results Using Euclidean } \\
\hline No of Samples & 5000 & 10000 & 20000 & 30000 & 40000 \\
\hline $\begin{array}{c}\text { No of clusters } \\
\text { formed }\end{array}$ & 83 & 122 & 145 & 116 & 189 \\
\hline Inter cluster & 4.6 & 4.8 & 5.13 & 6.89 & 6.989 \\
\hline $\begin{array}{c}\text { Average inter } \\
\text { cluster }\end{array}$ & 0.056 & 0.037 & 0.031 & 0.061 & 0.039 \\
\hline $\begin{array}{c}\text { Average Intra } \\
\text { cluster }\end{array}$ & 4.27 & 4.000 & 4.989 & 6.867 & 5.896 \\
\hline
\end{tabular}

CCA-Clustering Results Using Euclidean

\begin{tabular}{|c|c|c|c|c|c|}
\hline No of samples & 5000 & 10000 & 20000 & 30000 & 40000 \\
\hline $\begin{array}{c}\text { No of clusters } \\
\text { formed }\end{array}$ & 96 & 123 & 156 & 115 & 191 \\
\hline Inter cluster & 4.6 & 6.367 & 7.214 & 8.135 & 6.721 \\
\hline $\begin{array}{c}\text { Average Inter } \\
\text { cluster }\end{array}$ & 0.047 & 0.051 & 0.039 & 0.070 & 0.035 \\
\hline $\begin{array}{c}\text { Average Intra } \\
\text { cluster }\end{array}$ & 4.25 & 4.283 & 6.27 & 7.24 & 6.812 \\
\hline
\end{tabular}

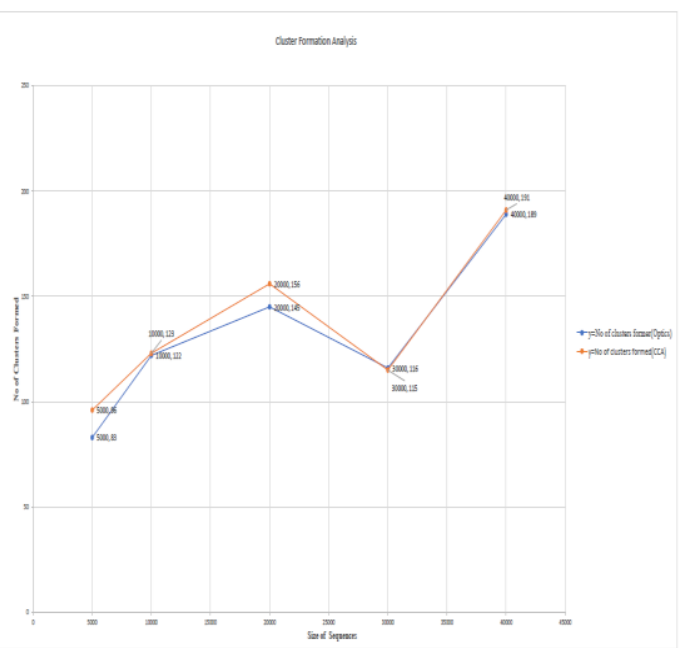

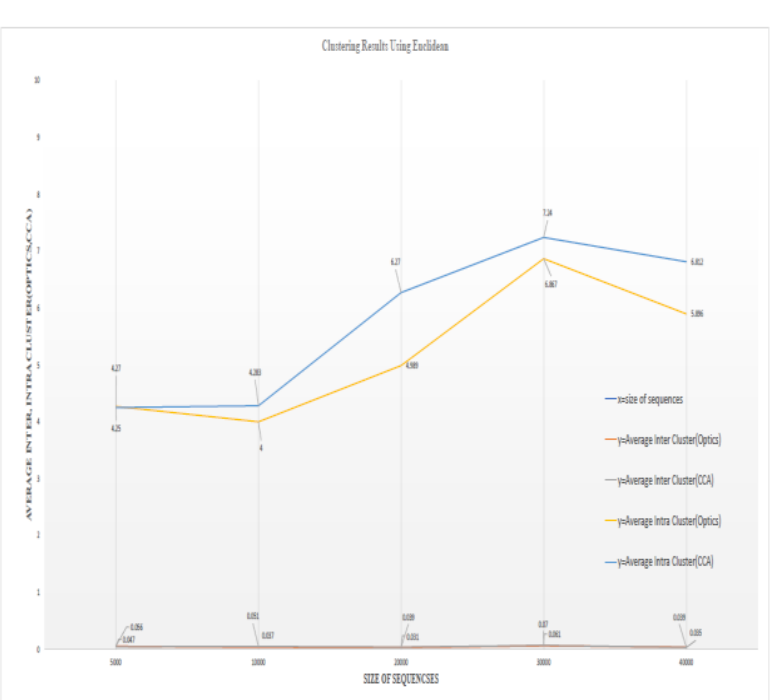

\section{TIME REQUIREMENTS}

Experiments were performed on the above mentioned dataset of varying sizes to see the performance of existing and proposed clustering algorithms. The number of clusters formed using by these for varying sizes of 5000,10000 , 20000, 30000 and 40000 transactions are recorded. The execution time taken for these varying sizes of samples are also recorded in table 6 .

Table 6. Time Requirements Of OPTICS and CCA

\begin{tabular}{|c|c|c|c|c|c|}
\hline \multicolumn{7}{|c|}{ OPTICS } \\
\hline $\begin{array}{c}\text { Size of } \\
\text { sequences }\end{array}$ & 5000 & 10000 & 20000 & 30000 & 40,000 \\
\hline No of clusters & 94 & 126 & 149 & 141 & 187 \\
\hline $\begin{array}{c}\text { Time taken in } \\
\text { seconds }\end{array}$ & 156 & 1879 & 3643 & 3218 & 4982 \\
\hline \multicolumn{7}{|c|}{ Enhanced ChameleonClustering Technique (CCA)using } \\
SSM \\
\hline $\begin{array}{c}\text { Size of } \\
\text { sequences }\end{array}$ & 5000 & 10000 & 20000 & 30000 & 40,000 \\
\hline $\begin{array}{c}\text { No of clusters } \\
\text { Nime taken in } \\
\text { seconds }\end{array}$ & 11444 & 1638 & 1064 & 1579 & 1555 \\
\hline
\end{tabular}

\section{CONCLUSIONS}

Considered arbitrarily web transactions from the MSNBC dataset and performed the experiments on Clustering algorithms.. We used previously existing/similarity measure namely Euclidean. For good clustering algorithm, the intra cluster distance should be minimum. Then using OPTICS and CCA, clusters are generated .Comparing OPTICS and CCA, the inter cluster similarity is maximum in CCA. For example in OPTICS for 5000 samples, the time taken for execution are $1156,3643,3218,4982$ respectively. The time taken to execute the algorithm CCA is less $(1144,1638,1064,1579,1555)$, when compare to other 
clustering techniques A variety of experiments are performed in the context of clustering on a sequential data in a web usage domain. This experiment shows that in addition to the content if Sequential Information is also added it improves thequality/accuracyofthe clustering. So Sequential information is important as well as Content information is also important.

\subsection{Future Work}

We extend our work in future to other clustering techniques and to other domains as well.

- The time complexities of the proposed algorithms can be improved further, which leads to better accuracy

\section{REFERENCES}

[1] Aggarwal.C, Han.J, Wang.J, Yu.P.S, "A Framework for Projected Clustering of High Dimensional Data Streams", 2004,pp.(852-863)Int. Conf. on Very Large Data Bases, Toronto, Canada.

[2] Aoying.Z, Shuigeng.Z, "Approaches for scaling DBSCAN algorithm to large spatial database", 2000,pp.(509-526),Journal of Computer Science and Technology, 15(6).

[3] Chen Song-Yu, O'Grady2,O'Hare, Wei Wang, "A Clustering Algorithm Incorporating Density and Direction", IAWTAC ,IEEE 2008.Deepak P, Shourya Roy IBM India Research Lab, OPTICS on Text Data:
Experiments and Test Results.

[4] Cooley.R,Mobasher, B,Srivastava.J, "Web mining: Information and pattern discovery on the world wide web", 9th IEEE Int. Conf. Tools AI.

[5] K.santhiSree, R.Kranthi Kumar, International Journal of computer publications:Case Study : Comparative Analysis: On Clustering of Sequential Data Streams USING Optics and ICA,2016,(34-37),135(2), (0975 8887).

[6] Guha.S, Mishra.N, Motwani.R, Callaghan.1,“ Clustering data streams". In Proceedings of Computer Science. IEEE,November,2000, pp(1391-1399), 16(10).

[7] K.Santhisree, Dr A.Damodaram, "SSM-DBSCAN and SSM-OPTICS : Incorporating a new similarity measure for Density based Clustering of Web usage data". 2011, International Journal on Computer Science and Engineering (IJCSE),.3(9),PP.(3170-3184)September India.

[8] K.Santhisree,"SSM-DENCLUE : Enhanced Approach for Clustering of Sequentialdata" Experiments and Test cases, June 2014.,International Journal of Computer Applications,96(6),pp.(7-14),Published by Foundation of Computer Science, New York, USA.

[9] https://wwwusers.cs.umn.edu/ hanxx023/dmclass/chameleon.pdf 